\section{Diagnostik der akuten Lungenembolie}

\author{
Empfehlungen der Deutsche Gesellschaft \\ für Pneumologie
}

E. Meissner (federführend), J. Niedermeyer, H. Worth, P. Dorow, S. Thalhofer, W. Petermann, H. W. Breuer, M. M. Borst, H. Olschewski, K. Rasche, R. Oertel, V. Sill

\section{Einleitung}

Akute Lungenembolien gehören zu den am häufigsten übersehenen Komplikationen bei internistischen und chirurgischen Patienten. Überträgt man Schätzungen der Inzidenz aus den USA, muss in der BRD mit fast 200000 Lungenembolien im Jahr gerechnet werden. Etwa 30000 verlaufen tödlich oder tragen wesentlich zum letalen Verlauf anderer Erkrankungen bei. Durch eine adäquate Therapie wird die Letalität der Lungenembolie (LE) von 30\% auf unter 8\% [1] gesenkt.

Das diagnostische Vorgehen richtet sich nach Schweregrad der LE sowie Verfügbarkeit der Diagnostik, sowohl apparativ als auch personell. Bereits beim klinischen Verdacht auf eine Lungenembolie muss mit therapeutischer Heparinisierung begonnen werden.

\section{Anamnese}

Eine Reihe von Risikofaktoren wie Immobilisierung, chirurgische Intervention, positive Familienanamnese u.a. werden bei Patienten mit LE gehäuft gegenüber einem gesunden Normalkollektiv gefunden. In der PIOPED-Studie [2] wurden Patienten $(n=365)$ prospektiv untersucht, die mit Verdacht auf LE in die Klinik aufgenommen wurden und bei denen im Verlauf bis hin zur Angiographie - eine LE ausgeschlossen $(n=248)$ oder nachgewiesen $(n=117)$ wurde. Es zeigten sich zwischen den beiden Gruppen zwar für Immobilisierung und chirurgische Interventionen hoch-signifikante Unterschiede, aber alle Risikofaktoren waren in beiden Gruppen nachweisbar (Tab.1). Ausschluss oder Nachweis einer LE anhand des Nachweises oder Fehlens von Risikofaktoren war nicht möglich.

Wichtig bei der Anamneseerhebung ist, an eine LE zu denken, nicht nur bei typischer Klinik, sondern z.B. auch bei flüchtigen Veränderungen wie Dyspnoe, Temperaturanstieg, Herzfrequenzanstieg, absoluter Arrhythmie bei Vorhofflimmern.

Nach hämostaseologischen Risikofaktoren wie Protein S-, Protein C- oder AT III-Mangel sowie insbesondere der Faktor V-Mutation muss gefragt werden.

Pneumologie 2000; 54: 587-591

(C) Georg Thieme Verlag Stuttgart · New York ISSN 0934-8387

\section{Klinik}

Für die von den Patienten angegebenen klinischen Symptome (Tab.1) wie Dyspnoe, pleurale Schmerzen, Beinschwellung, Beinschmerzen u.a., die an Beinvenenthrombose und Lungenembolie denken lassen, fanden sich in der PIOPED-Studie [2] keine signifikanten Unterschiede zwischen Patienten mit und ohne LE.

Bestimmte klinische Untersuchungsbefunde wie Tachypnoe, 4. Herzton, betonter 2. Herzton, Thrombosezeichen und Zyanose finden sich gehäuft bei Patienten mit LE im Vergleich mit einem Normalkollektiv. In der PIOPED-Studie [2] fanden sich zwar für Rasselgeräusche, 4. Herzton sowie betonten 2. Herzton signifikante Unterschiede zwischen Patienten mit und ohne LE (Tab. 1), alle klinischen Zeichen waren aber in beiden Gruppen nachweisbar.

LE-Nachweis oder -Ausschluss sind aufgrund einzelner klinischer Befunde ebenso wenig wie durch die Kombination anamnestischer Angaben und klinischer Befunde möglich [3].

\section{Labor}

Laborwerte haben keine diagnostische Aussagekraft hinsichtlich LE-Nachweis, sind aber wichtig für den Nachweis möglicher Differenzialdiagnosen.

Der Stellenwert der D-Dimer-Bestimmung ist bis heute nicht eindeutig geklärt. Der LE-Nachweis ist mit der Bestimmung nicht möglich - der Parameter ist zu unspezifisch. Unklar ist die Bedeutung beim LE-Ausschluss. Wichtig ist zunächst die Bestimmungsmethode des D-Dimerwertes. Nur ein ELISA-Test ist zuverlässig genug. Ein Latex-Agglutinations-Test sollte nicht verwendet werden. Auch mittels ELISA gemessen gibt es $5-10 \%$ falschnegative D-Dimerwerte [4], speziell dann, wenn eine Thrombose bereits mehrere Tage alt war, bevor sie zur Lungenembolie führt. Deshalb sollte bei dringendem klinischen Verdacht trotz negativem D-Dimerwert eine weiterführende Diagnostik durchgeführt werden [5]. Unklar ist bisher, ob die Vorhersagekraft bei geringem klinischen Verdacht und negativem D-Dimerwert zum LE-Ausschluss ausreicht. Insgesamt sind die vorliegenden Studien zu dieser Fragestellung noch nicht ausreichend [3].

Der Stellenwert einer Fibrinmonomer-Bestimmung in der LEDiagnostik ist zur Zeit nicht ausreichend evaluiert, um eine Aussage zu zulassen. 
Tab. 1 Prädisponierende Faktoren, Symptomatik und Untersuchungsbefunde bei Patienten $(n=365)$ ohne kardiopulmonale Vorerkrankungen mit V.a. Lungenembolie (mod. nach [2])

\begin{tabular}{|c|c|c|c|}
\hline & $\operatorname{LE}(n=117)$ & $\varnothing \operatorname{LE}(n=248)$ & \\
\hline \multicolumn{4}{|l|}{ prädisponierende Faktoren } \\
\hline Immobilisierung & $56 \%$ & $33 \%$ & $p<0,001$ \\
\hline chirurgischer Eingriff & $54 \%$ & $31 \%$ & $p<0,001$ \\
\hline Neoplasien & $23 \%$ & $15 \%$ & n.s. \\
\hline $\begin{array}{l}\text { Phlebothrombose } \\
\text { (anamnestisch) }\end{array}$ & $14 \%$ & $8 \%$ & n.s. \\
\hline $\begin{array}{l}\text { Trauma der unteren } \\
\text { Extremität }\end{array}$ & $10 \%$ & $10 \%$ & n.s. \\
\hline Östrogen-Einnahme & $9 \%$ & $10 \%$ & n.s. \\
\hline Schlaganfall & $7 \%$ & $4 \%$ & n.s. \\
\hline Postpartal-Zeit ( $\leq 3$ Monate) & $4 \%$ & $3 \%$ & n.s. \\
\hline \multicolumn{4}{|l|}{ Symptomatik } \\
\hline Dyspnoe & $73 \%$ & $72 \%$ & n.s. \\
\hline Pleuraschmerz & $66 \%$ & $59 \%$ & n.s. \\
\hline Husten & $37 \%$ & $36 \%$ & n.s. \\
\hline Beinschwellung & $28 \%$ & $22 \%$ & n.s. \\
\hline Beinschmerzen & $26 \%$ & $24 \%$ & n.s. \\
\hline Hämoptysen & $13 \%$ & $8 \%$ & n.s. \\
\hline Palpitationen & $10 \%$ & $18 \%$ & n.s. \\
\hline Giemen & $9 \%$ & $11 \%$ & n.s. \\
\hline Angina pectoris & $4 \%$ & $6 \%$ & n.s. \\
\hline \multicolumn{4}{|l|}{ Untersuchungsbefunde } \\
\hline Tachypnoe (> 20 min-1) & $70 \%$ & $68 \%$ & n.s. \\
\hline Rasselgeräusche & $51 \%$ & $40 \%$ & $p<0,05$ \\
\hline Tachykardie (> 100 min- 1 ) & $30 \%$ & $24 \%$ & n.s. \\
\hline 4. Herzton & $24 \%$ & $14 \%$ & $p<0,05$ \\
\hline betonter 2 . Herzton & $23 \%$ & $13 \%$ & $p<0,05$ \\
\hline Thrombosezeichen & $11 \%$ & $11 \%$ & n.s. \\
\hline Fieber $\left(>38,5^{\circ} \mathrm{C}\right)$ & $7 \%$ & $12 \%$ & n.s. \\
\hline Giemen & $5 \%$ & $8 \%$ & n.s. \\
\hline Pleurareiben & $3 \%$ & $2 \%$ & n.s. \\
\hline 3. Herzton & $3 \%$ & $4 \%$ & n.s. \\
\hline Zyanose & $1 \%$ & $2 \%$ & n.s. \\
\hline
\end{tabular}

n. s. = nicht signifikant

\section{EKG}

Die als typisch beschriebenen EKG-Veränderungen wie Rechtsverlagerung der Herzachse, P-pulmonale oder $\mathrm{S}_{\mathrm{I}} \mathrm{Q}_{\mathrm{III}}$-Typ sind nur in Ausnahmefällen und ggf. nur vorübergehend nachweisbar (Tab. 2). Wichtig ist das EKG in der Differenzialdiagnostik des Herzinfarktes.

\section{Lungenfunktion}

In der arteriellen Blutgasanalyse (BGA) beweist der für eine LE typische Befund - Hypoxämie trotz Hyperventilation $\left(\mathrm{P}_{\mathrm{a}} \mathrm{O}_{2} \downarrow\right.$, $\left.\mathrm{P}_{\mathrm{a}} \mathrm{CO}_{2} \downarrow\right)$ - keine LE, genauso wenig wie ein unauffälliger BGABefund eine LE ausschließt [3,5]. Das zeigen erneut die Daten der PIOPED-Studie [2]. Wichtig ist es, beim „typischen Befund“ an eine LE zu denken.
Tab. 2 EKG-Veränderungen bei Patienten mit akuter Lungenembolie ohne kardiopulmonale Vorerkrankungen ( $\mathrm{n}=89$; mod. nach [2])

\begin{tabular}{|c|c|}
\hline & Häufigkeit \\
\hline pathologisch & $70 \%$ \\
\hline Sinustachykardie & $90 \%$ \\
\hline $\begin{array}{l}\text { unspezifische ST-/T-Veränderungen, insbesondere } \\
\text { in rechts-präkordialen Ableitungen }\end{array}$ & $49 \%$ \\
\hline $\begin{array}{l}\text { P-pulmonale, RV-Hypertrophie, Rechtstyp, RSB, } \\
S_{1} Q_{I I I}\end{array}$ & $\leq 6 \%$ \\
\hline Vorhofflimmern & $4 \%$ \\
\hline SVES & $4 \%$ \\
\hline VES & $4 \%$ \\
\hline Vorhofflattern & $1 \%$ \\
\hline normal & $30 \%$ \\
\hline
\end{tabular}

Die häufig im Bereich der Intensivmedizin diskutierte $\mathrm{AaDO}_{2}$ weist gegenüber alleiniger BGA keine bessere Trennschärfe auf. In der PIOPED-Studie waren für die $\mathrm{AaDO}_{2}$ keine Unterschiede zwischen beiden Gruppen nachzuweisen [2]. Weder lässt eine erhöhte $\mathrm{AaDO}_{2}$ die Diagnose einer LE noch eine normale $\mathrm{AaDO}_{2}$ den Ausschluss einer LE zu [2].

Parameter der ventilatorischen Lungenfunktion zeigen keine richtungweisenden Veränderungen bei LE, allenfalls schmerzbedingt eine „Restriktion“.

\section{Röntgen-Thorax}

Wichtig ist die Röntgen-Thoraxaufnahme zur Erfassung von Differenzialdiagnosen wie z.B. Pneumothorax. Selten - insbesondere wenn Voraufnahmen in gleicher Technik bestehen können direkte Westermarksche Zeichen eine LE erkennen lassen. In der Regel haben Röntgen-Thoraxaufnahmen aber keinen beweisenden oder ausschließenden Charakter [3], wie die Daten der PIOPED-Studie (Tab. 3) gezeigt haben.

Bedeutung kann die Röntgen-Thoraxaufnahme in Kombination mit einer alleinigen Perfusions-Szintigraphie haben, um neben LE andere Ursachen von Perfusionsstörungen zu erkennen.

\section{Echokardiographie}

Die Echokardiographie ist als nicht-invasive Untersuchungsmethode am Patientenbett - auch auf Intensivstationen durchführbar. Bei hämodynamisch signifikanter LE ist sie eine wichtige Untersuchungsmethode (Tab. 4).

Bedeutsam ist der Nachweis einer akut aufgetretenen pulmonalen Hypertonie. Bei Trikuspidalklappeninsuffizienz kann der Druckgradient zwischen rechtem Vorhof und Ventrikel mittels modifizierter Bernoulli-Gleichung gemessen und durch Addition des geschätzten rechtsatrialen Drucks der systolische Druck im rechten Ventrikel errechnet werden. Selten ist ein direkter LE-Nachweis möglich. Der LE-Ausschluss ist nicht möglich, da bei hämodynamisch nichtsignifikanter LE typische Veränderungen fehlen. 
Tab. 3 Röntgenthoraxbefunde bei Patienten mit V.a. Lungenembolie (mod. nach [2])

\begin{tabular}{llll}
\hline & LE $(\mathrm{n}=117)$ & $\varnothing \mathrm{LE}(\mathrm{n}=248)$ \\
\hline Atelektase/Infiltrat & $68 \%$ & $48 \%$ & $\mathrm{p}<0,001$ \\
Pleuraerguss & $48 \%$ & $31 \%$ & $\mathrm{p}<0,01$ \\
pleuranahe Verschattung & $35 \%$ & $21 \%$ & $\mathrm{p}<0,01$ \\
Zwerchfellhochstand & $24 \%$ & $19 \%$ & $\mathrm{n} . \mathrm{s}$. \\
Gefäßrarifizierung & $21 \%$ & $12 \%$ & $\mathrm{p}<0,05$ \\
prominente zentrale & $15 \%$ & $11 \%$ & $\mathrm{n} . \mathrm{s}$. \\
Pulmonalgefäße & & & \\
Herzvergrößerung & $12 \%$ & $11 \%$ & $\mathrm{n} . \mathrm{s}$. \\
Gefäß-Kalibersprung & $7 \%$ & $2 \%$ & $\mathrm{n} . \mathrm{s}$. \\
Lungenödem & $4 \%$ & $13 \%$ & $\mathrm{p}<0,05$ \\
Normalbefund & $16 \%$ & $34 \%$ & $\mathrm{p}<0,001$ \\
\hline
\end{tabular}

n. s. = nicht signifikant

Tab. 4 Echokardiographische Befunde bei hämodynamisch bedeutsamer Lungenembolie

Rechter Ventrikel (RV)

- Zeichen der akuten rechtsventrikulären Druckbelastung

- akut druckbelasteter RV (allseits dilatiert)

- RV-Diameter in parasternaler Längsachse in Höhe der Mitralklappensegelspitzen $\rightarrow \geq 30 \mathrm{~mm}$ bei hämodynamisch wirksamer LE

- paradoxe Septumkontraktilität

- bis auf dyskinetisches Septum keine regional begrenzten Kontraktilitätsstörungen (DD: RV-Infarkt)

- DD: chronische Druck-/Volumenbelastung (RV-Wandhypertrophie)

- bei Trikuspidalinsuffizienz (Doppler-/Farbdoppler-Technik): Nachweis eines erhöhten systolischen PA-Drucks

Rechter Vorhof (RA)

- ggf. RA-Dilatation

- orientierend Abschätzung des RA-Druckes (V. cava inferiorDurchmesser)

- ggf. offenes Foramen ovale (paradoxe Embolien!)

Pulmonalarterie

- Dilatation des rechten Pulmonalishauptstammes

- evtl. direkter LE-Nachweis

Weitere Veränderungen

- Abnahme von linkskardialen Durchmessern

- ggf. Nachweis von intracavitären Thromben

- ggf. eingeschränkte Globalfunktion (durch Hypoxämie)

- verkürzte Akzelerationszeit zur Abschätzung eines erhöhten pulmonalarteriellen Drucks

Möglichkeiten im Rahmen der Therapie

- Therapiekontrolle: Rückbildung der Rechtsherzbelastung und ggf. Thrombusgefäße

\section{Venendiagnostik}

Die LE ist Komplikation einer Venenthrombose - in der Regel der tiefen Beinvenen. Angiologische Untersuchungen der tiefen Bein- und Beckenvenen [6] - auch bei klinisch unauffälligem Befund an den Beinen - sind als diagnostischer
Baustein wichtig, da bei einem Thrombosenachweis die LE wahrscheinlich wird [3]. Vorteil ist die Möglichkeit, bis auf die Phlebographie die Diagnostik - insbesondere die Duplexund Farbsonographie mit Kompression der Venen - am Patientenbett durchführen zu können. Die sonographischen Verfahren sind hinsichtlich des Nachweises einer Thrombose der oberflächlichen und tiefen Beinvenen bis zum Poplitealbereich der Phlebographie ebenbürtig.

Ein fehlender Thrombosenachweis lässt den Ausschluss einer LE nicht zu, da das gesamte thrombotische Material in die Pulmonalarterien verschleppt sein kann.

\section{Rechtsherzkatheter}

Mit dem Rechtsherzkatheter ist ähnlich der Echokardiographie eine Aussage über Vorliegen und Schweregrad einer pulmonalen Hypertonie möglich. Zusätzlich zur Echokardiographie können Herzzeitvolumen gemessen und pulmonaler Gefäßwiderstand berechnet werden.

Ein mittlerer pulmonalarterieller Druck von $\leq 18 \mathrm{mmHg}$ schließt eine LE nicht aus. Bei mittleren pulmonalarteriellen Druckwerten zwischen 20 und ca. $40 \mathrm{~mm} \mathrm{Hg}$ ist eine akute LE möglich. Mitteldruckwerte $\geq 40 \mathrm{mmHg}$ werden nur bei rezidivierender $\mathrm{LE}$, vorbestehender pulmonaler Hypertonie aus anderer Ursache oder vital bedrohlicher fulminanter LE gemessen.

Nachteil der Technik ist die erhöhte Komplikationsrate bei Thrombolysetherapie nach mehrfachen Punktionen oder Punktionsversuchen.

\section{Szintigraphie}

Der Wert der Methode wird häufig überschätzt. Die alleinige Perfusions-Szintigraphie ist nur zum LE-Ausschluss (Normalbefund) hilfreich. Nur der Befund mehrerer segmentaler oder lobulärer Perfusions-Ausfälle bei erhaltener Ventilation „hoch wahrscheinlicher“ Befund - ist als sicher zu werten (Tab. 5). Meistens - 73\% der Patienten in der PIOPED-Studie sind nur kombinierte Ventilations-Perfusions-Störungen nachweisbar, die ohne sichere Aussage sind und eine zusätzliche Diagnostik erfordern.

Tab. 5 Sensitivität und Spezifität der szintigraphischen Wahrscheinlichkeitsklassen gemessen an angiographischen Befunden [7]

\begin{tabular}{lll}
\hline Scan-Klasse & Sensitivität & Spezifität \\
\hline hohe Wahrscheinlichkeit & $41 \%$ & $97 \%$ \\
hohe, mittlere Wahrscheinlichkeit & $82 \%$ & $52 \%$ \\
hohe, mittlere, niedrige Wahrscheinlichkeit & $98 \%$ & $10 \%$ \\
\hline
\end{tabular}

\section{Spiral-Computertomographie (Angio-CT)}

Sicher ist mittels CT nur der Nachweis größerer Lungenembolien möglich. Im Bereich der zentralen Pulmonalarterien lassen sich Sensitivitäten und Spezifitäten von $95 \%$ bis $100 \%$ im Vergleich zur DSA erreichen. Durch den Einschluss der Segmentarterien sinkt nach den bisher vorliegenden Unter- 
suchungen die Sensitivität deutlich und wird mit teilweise nur $65 \%$ angegeben $[3,8,9]$. Subsegmental ist eine sichere Diagnose nicht mehr möglich [8]. Die guten Ergebnisse gelten nur für Spiral-CT-Geräte der neuesten Generation sowie ein entsprechendes angepasstes Untersuchungsprotokoll hinsichtlich Schichtdicke, Tischvorschub, KM-Bolus - Scanzeitpunkt (Delay) u.a. [10]. Ältere Geräte sind in der Wertigkeit deutlich schlechter. Wenn die Patienten die notwendige Apnoezeit von 10 Sekunden nicht einhalten können, wird die Aussage durch Bewegungsartefakte erschwert.

Vorteil der CT-Untersuchung gegenüber den bisher genannten Verfahren ist der direkte, nicht-invasiv geführte Nachweis von embolischem Material wie in der Angiographie. Weiterer Vorteil ist die Darstellung der gesamten Lunge mit dem Nachweis von Veränderungen, die z.B. zu pathologischen Befunden bei der Szintigraphie führen können. Zusätzlich lässt sich im Kontrastmittel-CT thrombotisches Material in V. cava inferior und Beckenvenen nachweisen, wenn die Bereiche mituntersucht werden.

\section{Kernspintomographie}

Mit der Kernspintomographie ist der Nachweis größerer Lungenembolien möglich. Im Vergleich mit dem Angio-CT sind Sensitivität und Spezifität schlechter. Gemessen an der Angiographie und begrenzt auf symptomatische Patienten fanden sich eine Sensitivität von $90 \%$ und eine Spezifität von $77 \%$ [11]. Ursache für die geringe Spezifität ist die fälschliche Interpretation von perihilärem und peribronchialem Fett als Embolie. Die Untersuchungen benötigen mehr Zeit als eine CT. Die Verfügbarkeit der Geräte ist geringer als die der Computertomographen.

\section{Digitale Subtraktionsangiographie (DSA)}

Nur im Bereich der großen Gefäße weist die DSA eine gleich hohe Sensitivität wie die konventionelle PulmonalarterienBlattfilmangiographien auf. Bei venöser DSA - KM-Injektion in periphere Vene - wird die gleiche KM-Menge benötigt und besteht das gleiche Risiko wie bei einer Blattfilmangiographie. Die direkte Injektion in die Pulmonalarterie erlaubt es, mit deutlich weniger Kontrastmittel bei DSA-Technik (ca. $20 \mathrm{ml}$ ) im Vergleich zur Blattfilmangiographie (ca. $50 \mathrm{ml}$ ) zu arbeiten.

Der Einsatz EKG-getriggerter Geräte ist hilfreich um Bewegungsartefakte insbesondere in Unterlappen zu vermeiden. Unklar ist bisher der Wert von C-Bogen mit DSA-Technik, die auf Intensivstationen eingesetzt werden können. Im Zusammenspiel mit durchleuchtungsfähigen Krankenbetten ist der DSA-Einsatz auf der Station im Bett möglich.

\section{Pulmonalis-Blattfilmangiographie}

Nach wie vor ist die konventionelle Blattfilmangiographie der Pulmonalarterien „golden Standard“ der Diagnostik zum Nachweis und Ausschluss der Lungenembolie. Aufgrund von hohem technischen Aufwand, langer Untersuchungsdauer, invasivem Charakter, Kontrastmittelrisiko - das durch die Verwendung nicht-ionischer KM reduziert werden kann -, sowie der Weiterentwicklung der Geräte wird die Technik zunehmend von der DSA verdrängt.

\section{Zusammenfassung}

Bei Verdacht auf Lungenembolie gibt es kein immer gültiges Standardvorgehen. Einfache diagnostische Maßnahmen (Abb.1) wie anamnestisch zu erhebende Risikofaktoren und Symptomatik, klinischer Untersuchungsbefund, Labor, EKG oder Röntgen-Thorax lassen in vielen Fällen weder Ausschluss noch Nachweis einer Lungenembolie zu.

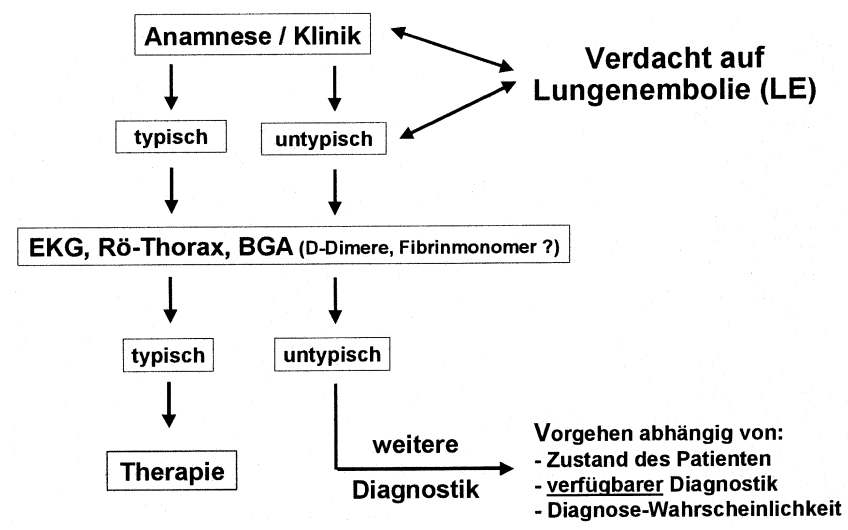

Abb. 1 Basis-Diagnostik bei V.a. Lungenembolie (modifiziert nach [12]).

Der weitere Ablauf der Diagnostik (Abb. 2) muss sich nach Zustand des Patienten und Verfügbarkeit der Diagnostik richten. Lange Patiententransporte zur weiteren Diagnostik z.B. zur Durchführung einer Ventilations-Perfusions-Szintigraphie in ein anderes Krankenhaus - sollten wegen der Gefahr eines möglicherweise letalen Rezidivs unterbleiben.

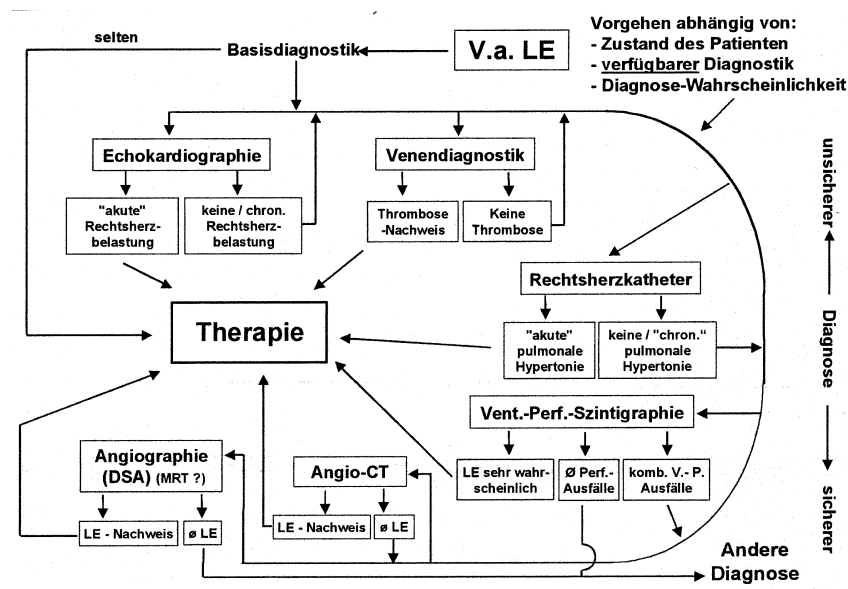

Abb. 2 Diagnostische Strategie bei V.a. Lungenemboile (modifiziert nach [12]).

Wegen der breiten Verfügbarkeit der sonographischen Methoden sollte zunächst versucht werden, eine LE über den Nachweis einer akut aufgetretenen pulmonalen Hypertonie mittels Echokardiographie oder einer tiefen Beinvenenthrombose mittels Farbdoppler-Sonographie wahrscheinlich zu machen. Wenn diese Untersuchungen keine wegweisende Aussage ermöglichen, ist ein bildgebendes Verfahren (Szinti- 
graphie, Angio-CT) anzustreben. Das Angio-CT gewinnt hier eine zunehmende Bedeutung, da es nicht-invasiv einen LENachweis zulässt. Die CT lässt neben der Beurteilung der Lungengefäße zusätzlich andere Lungenveränderungen erkennen, die in der Szintigraphie zu pathologischen Befunden führen können. Im Zweifelsfall muss nach wie vor mit der Pulmonalisangiographie - in DSA-Technik oder konventioneller Blattfilmtechnik - die Diagnose gesichert oder ausgeschlossen werden.

\section{Literatur}

${ }^{1}$ Dalen JE, Alpert JS. Natural history of pulmonary embolism. Prog Cardiovasc Dis 1975; 17: 259-270

2 Stein PD, Terrin ML, Hales CA, Palevsky HI, Saltzman HA, Thompson BT, Weg JG. Clinical, laboratory, roentgenographic, and electrocardiographic findings in patients with acute pulmonary embolism and no pre-existing cardiac or pulmonary disease. Chest 1991; 100: 598-603

${ }^{3}$ Tapson VF, Carroll BA, Davidson BL, Elliott CG, Fedullo PF, Hales CA, Hull RD, Hyers TM, Leeper Jr KV, Morris TA, Moser KM, Raskob GE, Shure D, Sostman HD, Taylor Thompson B. The diagnostic approach to acute venous thromboembolism. Clinical practice guideline. American Thoracic Society. Am J Respir Crit Care Med 1999; 160: 1043 - 1066

${ }^{4}$ Bounameaux H, Perrier A. Role of D-Dimers in the exclusion of pulmonary embolism. Sem Respir Crit Care Med 1996; 17: 31 37

${ }^{5}$ ACCP Consensus Committee on Pulmonary Embolism. Opinions regarding the diagnosis and management of venous thromboembolic disease. Chest 1996; 109: 233-237

${ }^{6}$ Blättler W, Partsch H, Hertel T. Leitlinie zur Diagnostik und Therapie der tiefen Bein-/Beckenvenenthrombose. Phlebologie 1998; 27: $84-88$

${ }^{7}$ PIOPED-Investigators. Value of the ventilation/perfusion scan in acute pulmonary embolism. Results of the prospective investigation of pulmonary embolism diagnosis (PIOPED). JAMA 1990; 263: $2753-2759$

${ }^{8}$ ACCP Consensus Committee on Pulmonary Embolism. Opinions regarding the diagnosis and management of venous thromboembolic disease. Chest 1998; 113: 499-504

${ }^{9}$ Mullins MD, Becker DM, Hagspiel KD, Philbrick JT. The role of spiral volumetric computed tomography in the diagnosis of pulmonary embolism. Arch Intern Med 2000; 160: 293-298

${ }^{10}$ Remy-Jardin M, Remy J. Spiral CT angiography of the pulmonary circulation. Radiology 1999; 212: 615-636

${ }^{11}$ Erdman WA, Peshock RM, Redman HC, Bonte F, Meyerson M, Jayson HT, Miller GL, Clarke GD, Parkey RW. Pulmonary embolism: comparison of MR images with radionuclide and angiographic studies. Radiology 1994; 190: 499-508

${ }^{12}$ Meissner E, Niedermeyer J, Fabel H. Akute Lungenembolie. Z Kardiol 1993; 2: 3-12

\section{Dr. E. Meissner}

Abteilung Pneumologie

Medizinische Hochschule Hannover

Carl-Neuberg-Straße 1

30625 Hannover

E-mail: eckehard.meissner@t-online.de

\section{Zweites Deutsch-Rumänisches}

Pneumologiesymposium

Am 25. und 26. September 2000 fand in Bukarest das 2. zweitägige deutsch-rumänische Symposium statt. Es handelt sich dabei um einen postgraduierten Kurs, der von Vertretern beider wissenschaftlicher Gesellschaften, also der Deutschen Gesellschaft für Pneumologie und der Rumänischen Gesellschaft für Pneumologie beschickt wird. Von deutscher Seite nahmen die Professoren Dierkesmann, Gillessen, Konietzko und Morr teil, von rumänischer Seite die Professoren Bruckdan, Mihaltan und Stoikesjcu. Die Thematik erstreckte sich von ambulant erworbener Pneumonie über obstruktive Atemwegserkrankung, Lungenkrebs und interstitielle Lungenerkrankung bis hin zu asbestassoziierten pleuropulmonalen Erkrankungen.

Die Kurse fanden unter Schirmherrschaft der Deutschen Botschaft im Goethe-Institut in Bukarest statt und waren durchgehend von über 100 Pneumologen aus ganz Rumänien besucht. Wegen des großen Echos und des nach wie vor vorhandenen Bedarfs an wissenschaftlicher und praktisch klinischer Kommunikation ist die Fortsetzung dieser Treffen in zweijährigem Turnus auch in Zukunft geplant. 